\title{
Comparison of iodine intake and status of UK vegans, vegetarians, and omnivores in 2016-17 and 2019
}

\author{
ER. Eveleigh ${ }^{1}$, LJ. Coneyworth ${ }^{1}$ and SJM. Welham ${ }^{1}$ \\ ${ }^{1}$ Division of Food, Nutrition \& Dietetics, School of Biosciences, The University of Nottingham, \\ Sutton Bonington, Leicestershire, UK
}

Iodine is required for thyroid hormone synthesis ${ }^{(1)}$. The UK was considered iodine sufficient until recently. Iodine- fortification policies are not implemented nationally, therefore, adequate intake is dependent on iodine rich food consumption ${ }^{(2)}$. Dairy products, white fish and eggs are the primary sources of iodine in the UK diet. Individuals following vegan and vegetarian diets may be vulnerable to deficiency due to not consuming typical sources of iodine. Few studies exist addressing iodine in these diets, and indicators of iodine nutrition have not been monitored in these groups across time ${ }^{(3)}$. The aim of the study was to compare the iodine nutrition of UK vegans and vegetarians with omnivores, assess agreement to recommended intake (RI) of $150 \mu \mathrm{g}$ day-1 for iodine, and to monitor iodine intake and status between years 2016-17 and 2019.

Dietary intake ( $\mu$ g day-1) was estimated using three-day food diaries (FD), and iodine specific food frequency questionnaires (FFQ). Urinary iodine concentration (UIC) was used to assess status and was analysed by ICP-MS according to WHO criteria. IBM SPSS was used for statistical analysis. Normality was assessed and non-parametric tests employed.

Nighty-five students and staff of The University of Nottingham (aged 18-60 years) were recruited between October 2016- 17 and June 2019. Median iodine intake recorded was below the RI. Vegan and vegetarian groups had the lowest iodine intake, which was significantly lower in vegans compared to omnivores in $2019(\mathrm{P}<0.001)$ but not $2016-17(\mathrm{P}=0.132)$. UIC was greater in omnivores (2016-17; $79.4 \mu \mathrm{g} \mathrm{L}-1 ., 2019 ; 72.4 \mu \mathrm{g} \mathrm{L}-1)$, than vegetarians (2016-17; $32.7 \mu \mathrm{g} \mathrm{L}-1 ., 2019$;

$13.4 \mu \mathrm{g} \mathrm{L}-1)$ and vegans, who had the lowest concentration (2016-17; 31.2 $\mu \mathrm{g} \mathrm{L}-1 ., 2019 ; 12.2 \mu \mathrm{g} \mathrm{L}-1)$. In 2016-17, omnivores had significantly higher UIC than vegans $(\mathrm{P}=0.038)$ but not vegetarians $(\mathrm{P}=0.796)$. Significance was not present in 2019 between omnivores and vegans $(\mathrm{P}=0.084)$ or vegetarians $(\mathrm{P} 1.000)$.

Our findings confirm that vegans and vegetarians are at risk of inadequate iodine nutrition across time and suggest that iodine in the UK diet is still an issue regardless of dietary preference. Further monitoring of these groups is needed, particularly given the rise in popularity of these diets. Comprehensive investigation into improving iodine intake and status in individuals adhering to vegan and vegetarian diets is required.

\section{References}

1. Zimmermann M (2009) Endocr. Rev 30, 376-408.

2. Bath SC, Button S \& Rayman MP (2014) Public Health Nutr 2, 450-4.

3. Eveleigh ER, Coneyworth LJ, Avery A, et al. (2020) Nutrients 12, 1606.

4. Zimmermann MB, Andersson M (2012) Nutr Rev 70, 553-70. 